\title{
A Statistical Measure of Global Equity Market Risk
}

\author{
Daniel Felix Ahelegbey \\ Department of Economics and Management, University of Pavia, Pavia, Italy \\ Email: danielfelix.ahelegbey@unipv.it
}

How to cite this paper: Ahelegbey, D.F. (2020) A Statistical Measure of Global Equity Market Risk. Applied Mathematics, 11, 1053-1060.

https://doi.org/10.4236/am.2020.1111070

Received: October 17, 2020

Accepted: October 31, 2020

Published: November 3, 2020

Copyright $\odot 2020$ by author(s) and Scientific Research Publishing Inc. This work is licensed under the Creative Commons Attribution International License (CC BY 4.0).

http://creativecommons.org/licenses/by/4.0/

\begin{abstract}
We construct a new index of global equity market risk (EMR) using market interconnectedness and volatilities. We study the relationship between our EMR and the VIX over the last two decades. The EMR is shown to be a novel approach to measuring global market risk, and an alternative to the VIX. Using data of 20 major stock markets, including G10 economies, we find spikes in our EMR index during the dotcom bubble, the global financial crisis, the European sovereign debt crisis, and the novel coronavirus pandemic. The result shows that the global financial crisis and the COVID-19 induced crisis record the historic highest spikes in financial market risk, suggesting stronger evidence of contagion in both periods.
\end{abstract}

\section{Keywords}

COVID-19, Financial Crises, Financial Markets, Market Risk, Mahalanobis Distance, Volatility Index

\section{Introduction}

Concerns about monitoring financial market turbulence and risk have intensified in recent times, especially in the heat of the ongoing global pandemic. The reaction of investors and financial markets since the first quarter of 2020, following the spread of the novel coronavirus (COVID-19) from Wuhan, in China to a global pandemic, has heightened the need to find ways of tracking the effect of the COVID-19 induced crisis on financial markets. This paper proposes the construction of a new index of equity market risk (EMR) using market interconnectedness and volatilities. The latter is considered a measure of market uncertainty or fear, which can be proxied via standard deviation of returns. The interconnectedness among markets provides the channels for spillover propagation. 
Our approach to the construction of the EMR follows the Mahalanobis turbulence measure of [1], and it is distinct from the systemic risk measures in [2]-[8]. The closest benchmark to our measure is the VIX - the Chicago Board of Exchange volatility index, generally used for measuring the level of global market risk.

We study the relationship between our EMR index and the VIX by using daily prices of 20 major stock market from Bloomberg, covering January 2000 to June 2020. The result shows spikes in our EMR index during the dotcom bubble, the global financial crisis, the European sovereign debt crisis, and the novel coronavirus pandemic. We find evidence of a significant relationship between the EMR and the VIX, with both indices providing similar signals about the direction of global market risk.

The organization of the paper is as follows: Section 2 presents the methodology; Section 3 reports the results; and Section 4 concludes the paper.

\section{Methodology}

In this section, we briefly present the background to network models. Next, we describe our measure of equity market risk (EMR), and a simple model of the relationship between the EMR and the VIX.

\subsection{Modeling Interconnectedness}

We model interconnectedness among markets via network models. A network model is a convenient class of multivariate analysis that uses graphs to represent statistical models [9]. They are formally represented by $(G, \theta) \in(\mathcal{G} \times \Theta)$, where $G$ is a graph of relationships between variables, $\theta$ is the model parameter, $\mathcal{G}$ is the space of graphs and $\Theta$ is the parameter space. The graph, $G$, is defined by a set of vertices (nodes/variables) joined by a set of edges (links), describing the statistical relationships between a pair of variables. A typical multivariate multiple regression model is given by

$$
Y=B X+U
$$

where $X=\left(X_{1}, \cdots, X_{n}\right)$ and $Y=\left(Y_{1}, \cdots, Y_{n}\right)$ are vector of exogenous and response variables respectively, $B$ is a coefficient matrix and $U$ is a vector of errors typically assumed to be multivariate normal. In this example, relationships between $X$ and $Y$ can be summarized by a weighted, $A^{w} \in \mathbb{R}^{n \times n}$, or unweighted adjacency matrix, $A \in\{0,1\}^{n \times n}$, whose $i j$-th element

$$
A_{i j}=\left\{\begin{array}{ll}
0, & \text { if } G_{i j}=0, X_{j} \rightarrow Y_{i} \\
1, & \text { otherwise, } X_{j} \rightarrow Y_{i}
\end{array}, A_{i j}^{w}= \begin{cases}0, & \text { if } B_{i j}=0 \\
B_{i, j} & \text { otherwise }\end{cases}\right.
$$

where $X_{j} \rightarrow Y_{i}$ means $X_{j}$ influences $Y_{i}$, and $A_{i j}^{w}$ specifies the weight of the relationship.

\subsection{Measuring Equity Market Risk (EMR)}

Let $Y=\left(Y_{1}, \cdots, Y_{n}\right)$ be return series of $n$-assets with risks $\sigma_{Y}=\left(\sigma_{1}, \cdots, \sigma_{n}\right)$ and let $A^{w}$ be a weighted adjacency matrix. Assume $S_{\sigma}=\sigma_{Y} \sigma_{Y}^{\prime}$ is the inner-product 
of risks, and $\Omega=\left(I+A^{w}\right)^{\prime}\left(I+A^{w}\right)$ is their constrained precision matrix. Following [1] and [10], we construct an index of equity market risk (EMR) as:

$$
\mathrm{EMR}=\frac{1}{n} \operatorname{tr}\left(\Omega S_{\sigma}\right)
$$

where EMR captures the average degree of unusual changes in asset returns and their interactions. The index signals widespread market turmoil and can be viewed as a measure of market-level fears (volatilities) that are amplified through interconnectedness.

The above index uses market interconnectedness $\left(A^{w}\right)$ and volatilities $\left(S_{\sigma}\right)$. The latter can be estimated directly from time series of stock market price/returns. The market interconnectedness measure $\left(A^{w}\right)$ is supposed to capture weighted channels of influence among markets. Thus, $A^{w}$ can be estimated by approximating the dynamics in observed multivariate financial time series as in (1) and (2). The commonly discussed methods for estimating $A^{w}$ are: Granger-causality [5]; Lasso regularization [11] [12]; forecast error variance decomposition [6]; and Bayesian graph structural learning [13] [14] [15]. In this study, we derive the weighted $A^{w}$ via a Bayesian graph structural learning approach as in [10] [14].

\subsection{Modeling Relationship between EMR and VIX}

We model the marginal distribution of the EMR as ARIMA $(1,1,1)$ and the conditional distribution of the VIX given $\operatorname{EMR}$ as $\operatorname{ARIMAX}(2,1,1)$.

$$
\begin{gathered}
\Delta \mathrm{EMR}_{t}-\Phi_{1} \Delta \mathrm{EMR}_{t-1}+\Theta_{1} \xi_{t-1}^{e}+\xi_{t}^{e} \\
\Delta \mathrm{VIX}_{t}=\beta \Delta \mathrm{EMR}_{t}+\Phi_{1} \Delta \mathrm{VIX}_{t-1}+\Phi_{2} \Delta \mathrm{VIX}_{t-2}+\Theta_{1} \xi_{t-1}^{v}+\xi_{t}^{v} \\
\Delta \mathrm{VIX}_{t}=\Phi_{1} \Delta \mathrm{VIX}_{t-1}+\Theta_{1} \xi_{t-1}^{v}+\xi_{t}^{v}
\end{gathered}
$$

where $\Delta \mathrm{EMR}_{t}$ is the first difference of equity market risk index at time $t$, $\Delta$ VIX $_{t}$ is the first difference of the VIX index at time $t, \xi_{t}^{e}$ is the error term of the EMR model, and $\xi_{t}^{v}$ is the error term of the VIX model. $\Phi_{1}$ is a first-order autoregressive (AR) term, while $\Theta_{1}$ is a first-order moving average (MA) term, and $\beta$ captures the contemporaneous relationship between EMR and VIX. Thus, if $\beta$ is significantly different from zero then EMR has significant information to improve the prediction of the VIX. The above model specification is such that (4) is the ARIMA $(1,1,1)$ EMR model, (5) is the $\operatorname{ARIMAX}(2,1,1)$ VIX model with the EMR as an exogenous variable, and (6) is the ARIMA $(1,1$, 1) VIX benchmark model.

\section{Empirical Results}

\subsection{Data Description}

We study the relationship between our EMR index and the VIX by using data from the Bloomberg database. The data consists of the daily market indices of 20 countries, including G10 economies, covering January 3, 2000, to June 30, 2020. We consider only one index per country, which typically contains the stock 
prices of the largest companies listed in the nation's largest stock exchange. The considered countries can be grouped into three regions: the Americas (Brazil, Canada, Mexico, and the United States), Asia-Pacific (Australia, China, Hong Kong, India, Japan, and South Korea), and Europe (Belgium, France, Germany, Italy, the Netherlands, Portugal, Russia, Spain, Switzerland, and the United Kingdom). A description of the market indices chosen for the selected countries is presented in Table 1.

The selected market indices vary in terms of composition, in the sense that some have a smaller number of stocks compared to others. For instance, the U.S. is represented by the S \& P 500, which contains the stocks of the top 500 large-cap corporations, whereas France is represented by CAC 40, which contains 40 stocks selected among the top 100 corporations.

Figure 1 reports the plot of daily closing prices on a logarithmic scale. Due to differences in the values, plotting the original prices would be difficult to visualize. We, therefore, scale the prices to a zero mean and unit variance and add the absolute minimum value of each series to avoid negative outcomes. This standardizes the scale of measurement for the different series. The plot shows that, amid many fluctuations, and some local specificities, stock markets are highly synchronized and have been affected by four major financial stresses characterized by huge drop in prices. These major financial stress periods are: 1) the tech-bubble crisis of 2000-2003 which was triggered by an endogenous shock fuelled via the adoption of the internet in the late 1990's, especially in developed markets; 2) the sub-prime and global financial crisis of 2007-2009 which was triggered by the massive defaults of sub-prime borrowers in the US mortgage market; 3) the European sovereign debt crisis of 2010-2013 which emanated from the inability of a cluster of EU member states to repay or refinance their sovereign debt and bailout heavily leveraged financial institutions without recourse to third party assistance; and 4) the ongoing distress to the world economy and global financial markets caused by the novel coronavirus pandemic in 2020.

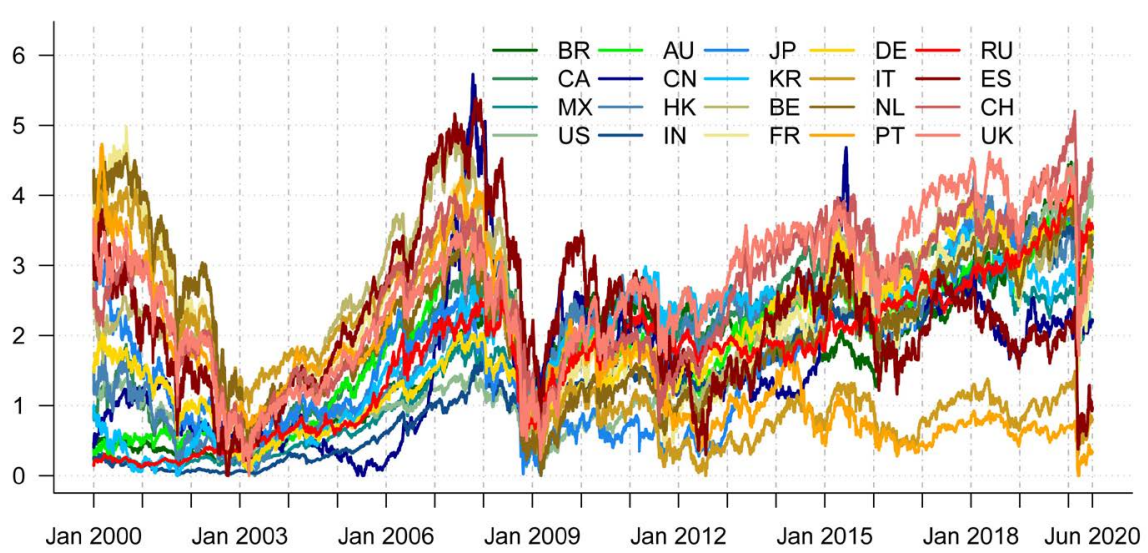

Figure 1. Time series of daily stock market prices on a logarithmic scale (January 3, 2000-June 30, 2020). 
Table 1. Detailed description of stock market indices of countries classified according to regions.

\begin{tabular}{|c|c|c|c|c|c|}
\hline Region & No. & Country & Code & Description & Index \\
\hline \multirow[t]{4}{*}{ Americas } & 1 & Brazil & $\mathrm{BR}$ & Brazil Bovespa & IBOV \\
\hline & 2 & Canada & CA & Canada TSX Comp. & SPTSX \\
\hline & 3 & Mexico & MX & Mexico IPC & MEXBOL \\
\hline & 4 & United States & US & United States S \& P 500 & SPX \\
\hline \multirow[t]{6}{*}{ Asia-Pacific } & 5 & Australia & $\mathrm{AU}$ & Australia ASX 200 & AS51 \\
\hline & 6 & China & $\mathrm{CN}$ & China SSE Comp. & SHCOMP \\
\hline & 7 & Hong Kong & $\mathrm{HK}$ & Hong Kong Hang Seng & HSI \\
\hline & 8 & India & IN & India BSE Sensex & SENSEX \\
\hline & 9 & Japan & JP & Japan Nikkei 225 & $\mathrm{NKY}$ \\
\hline & 10 & Korea & $\mathrm{KR}$ & South Korean KOSPI & KOSPI \\
\hline \multirow[t]{10}{*}{ Europe } & 11 & Belgium & $\mathrm{BE}$ & Belgium BEL 20 & BEL20 \\
\hline & 12 & France & FR & France CAC 40 & CAC \\
\hline & 13 & Germany & $\mathrm{DE}$ & Germany DAX 30 & DAX \\
\hline & 14 & Italy & IT & Italy FTSE MIB & FTSEMIB \\
\hline & 15 & Netherlands & NL & Netherlands AEX & AEX \\
\hline & 16 & Portugal & PT & Portugal PSI 20 & PSI20 \\
\hline & 17 & Russia & RU & Russia MOEX & IMOEX \\
\hline & 18 & Spain & ES & Spain IBEX 35 & IBEX \\
\hline & 19 & Switzerland & $\mathrm{CH}$ & Switzerland SMI & SMI \\
\hline & 20 & United Kingdom & UK & UK FTSE 100 & UKX \\
\hline
\end{tabular}

\subsection{Results}

We compute daily returns as the log differences of successive daily closing prices. We obtain monthly estimates of the model parameters and construct the matrices $\Omega$ and $S_{\sigma}$, which are the core components of our EMR index. To improve the efficiency of the estimates of $\Omega$ we aggregate monthly estimates in yearly rolling windows of about 240 trading days. We set the increments between successive rolling windows to one month, setting the first window of our study from February 1, 1999, to January 31, 2000, followed by March 1, 1999, to February 29, 2000; the last window is from July 1, 2019, to June 30, 2020. In total, we consider 246 rolling windows. To avoid over smoothing, $S_{\sigma}$ is instead estimated monthly, that is, using only the last month of each rolling window.

We report in Figure 2 the plot of the EMR and the VIX. The figure shows a striking resemblance between the EMR and VIX, both proving a similar signal about the "direction" of global market risk. Both indices indicate spikes during the tech-bubble crisis (2000-2003), the global financial crisis (GFC, 2007-2009), the Eurozone crisis (2010-2013), the Chinese stock market turbulence (2015-2016), 
and the recent COVID-19 pandemic (2020: 1H). The historical highest spike recorded for the COVID is only second to that of the GFC, which indicates evidence of strong contagion in the equities market during the GFC and the COVID pandemic, more than in the tech-bubble and Eurozone crisis. This suggests that the behavior of the stock markets during the pandemic is in several ways more similar to the GFC than it is to other periods of financial market stress.

We present in Table 2 the cross-correlation of the first difference of VIX with EMR. The table reports the highest cross-correlation occurring at lag 0 , which suggests evidence of a strong positive contemporaneous relationship. Table $3^{1}$ confirms the significant contemporaneous impact of the EMR on the VIX. Table 4 presents the out-of-sample forecast of the monthly VIX for the rest of the year 2020 compared with the realized values for the months of July-September $2020^{2}$. The results indicate that not only does the $\operatorname{ARIMAX}(2,1,1)$ performs better than the benchmark-ARIMAX $(1,1,1)$ in terms of the RMSE of the in-sample training set, but also the out-sample forecasts are much closer to the realized observation than the benchmark. In summary, we document evidence of a significant relationship between the EMR and VIX, with both indices providing similar signals about the direction of global market risk. In addition, the EMR improves the prediction of the VIX.

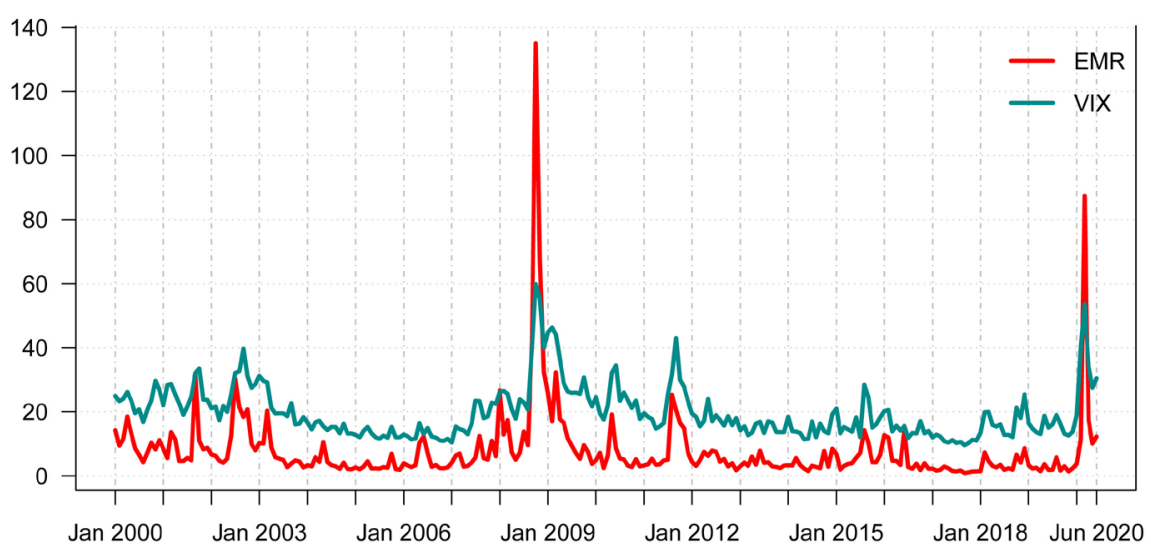

Figure 2. Historic monthly series of EMR and VIX (January 2000-June 2020).

Table 2. Cross-correlation of VIX with EMR. The result shows the correlation of $x_{t+h}$ (the row variable) with $y_{t}$ (the column variable). Boldface values indicate the significant correlations.

\begin{tabular}{cccccccccc}
\hline \multicolumn{10}{c}{ Cross-correlation of $\Delta \operatorname{VIX}(\mathrm{t})$ with } \\
\hline$x_{t+h}$ & -4 & -3 & -2 & -1 & 0 & 1 & 2 & 3 & 4 \\
$\Delta$ EMR & 0.024 & 0.016 & -0.247 & 0.042 & $\mathbf{0 . 5 8 0}$ & 0.123 & -0.265 & -0.135 & 0.006 \\
\hline
\end{tabular}

${ }^{1}$ We conduct model diagnostics to ensure there is no serial correlation and heteroscedasticity in residuals.

${ }^{2}$ We forecast out-of-sample EMR which are then used for predicting the VIX via $\operatorname{ARIMAX}(2,1,1)$. 
Table 3. Impact of EMR on VIX. Note: ${ }^{* *}$ and ${ }^{* *}$ are $1 \%$ and $5 \%$ level of significance respectively.

\begin{tabular}{cccc}
\hline & \multicolumn{2}{c}{ EMR } & \\
\cline { 2 - 4 } & ARIMA $(1,1,1)$ & $\operatorname{ARIMAX}(1,1,1)$ & $\operatorname{ARIMA}(1,1,1)$ \\
\hline$\Phi_{1}$ & $0.5547^{* * *}$ & $0.4690^{* * *}$ & $0.7601^{* * *}$ \\
$\Phi_{2}$ & & $0.2008^{* *}$ & \\
$\Theta_{1}$ & $-0.9999^{* * *}$ & $-0.8949^{* * *}$ & $-0.9328^{* * *}$ \\
$\beta$ & & $0.3078^{* * *}$ & \\
$\sigma^{2}$ & 102.0953 & 13.2295 & 21.4432 \\
AIC & 1838.8690 & $1,338.3600$ & 1452.7390 \\
RMSE & 10.0837 & 3.6298 & 4.6213 \\
\hline
\end{tabular}

Table 4. Out-of-sample point forecast of VIX according to $\operatorname{ARIMAX}(2,1,1)$ and ARIMA $(1,1,1)$.

\begin{tabular}{lccc}
\hline & & \multicolumn{2}{c}{ Forecast (VIX) } \\
\cline { 3 - 4 } & Realized (VIX) & ARIMAX $(2,1,1)$ & ARIMA $(1,1,1)$ \\
\hline $2020-07$ & 24.4600 & 27.9478 & 28.8076 \\
$2020-08$ & 26.4100 & 27.1818 & 27.5744 \\
$2020-09$ & 26.3700 & 26.4143 & 26.6371 \\
$2020-10$ & & 25.9505 & 25.9247 \\
$2020-11$ & & 25.6066 & 25.3832 \\
$2020-12$ & & 25.3676 & 24.9716 \\
\hline
\end{tabular}

\section{Conclusion}

This paper constructs an index of equity market risk (EMR) using market interconnectedness coupled with individual volatilities. The EMR index is shown to be a novel approach to measuring global market risk, and an alternative to the VIX. The empirical application presents a study of the relationship between the EMU and the VIX over the last 20 years. We find evidence of a significant relationship between both indices providing similar signals about the direction of global market risk. The result shows spikes in both indices during the dotcom bubble, the global financial crisis, the European sovereign debt crisis, and the novel coronavirus pandemic. However, the spikes recorded during the global financial crisis and the COVID-19 induced crisis suggest stronger evidence of financial stress and market tension in both periods.

\section{Acknowledgements}

We would like to thank the editor and referee for their comments on an earlier version of this paper. We have benefited immensely from the support of Paolo Giudici. Authors' research is supported by Funding from the European Union's 
Horizon 2020 research and innovation programme under grant agreement No 825215 (Topic: ICT-35-2018 Type of action: CSA).

\section{Conflicts of Interest}

The author declares no conflicts of interest regarding the publication of this paper.

\section{References}

[1] Kritzman, M. and Li, Y. (2010) Skulls, Financial Turbulence, and Risk Management. Financial Analysts Journal, 66, 30-41. https://doi.org/10.2469/faj.v66.n5.3

[2] Adrian, T. and Brunnermeier, M.K. (2016) CoVaR. The American Economic Review, 106, 1705-1741. https://doi.org/10.1257/aer.20120555

[3] Kritzman, M., Li, Y., Page, S. and Rigobon, R. (2011) Principal Components as a Measure of Systemic Risk. Journal of Portfolio Management, 37, 112. https://doi.org/10.3905/jpm.2011.37.4.112

[4] Huang, X., Zhou, H. and Zhu, H. (2012) Systemic Risk Contributions. Journal of Financial Services Research, 42, 55-83. https://doi.org/10.1007/s10693-011-0117-8

[5] Billio, M., Getmansky, M., Lo, A.W. and Pelizzon, L. (2012) Econometric Measures of Connectedness and Systemic Risk in the Finance and Insurance Sectors. Journal of Financial Economics, 104, 535-559. https://doi.org/10.1016/j.jfineco.2011.12.010

[6] Diebold, F.X. and Yilmaz, K. (2014) On the Network Topology of Variance Decompositions: Measuring the Connectedness of Financial Firms. Journal of Econometrics, 182, 119-134. https://doi.org/10.1016/j.jeconom.2014.04.012

[7] Brownlees, C. and Engle, R.F. (2017) SRISK: A Conditional Capital Shortfall Measure of Systemic Risk. The Review of Financial Studies, 30, 48-79. https://doi.org/10.1093/rfs/hhw060

[8] Banulescu, G.-D. and Dumitrescu, E.-I. (2015) Which Are the SIFIs? A Component Expected Shortfall Approach to Systemic Risk. Journal of Banking and Finance, 50, 575-588. https://doi.org/10.1016/j.jbankfin.2014.01.037

[9] Lauritzen, S.L. (1996) Graphical Models. Oxford University Press, Oxford.

[10] Ahelegbey, D.F. and Giudici, P. (2020) Market Risk, Connectedness and Turbulence: A Comparison of 21st Century Financial Crises. https://doi.org/10.2139/ssrn.3584510

[11] Kock, A.B. and Callot, L. (2015) Oracle Inequalities for High Dimensional Vector Autoregressions. Journal of Econometrics, 186, 325-344.

https://doi.org/10.1016/j.jeconom.2015.02.013

[12] Basu, S. and Michailidis, G. (2015) Regularized Estimation in Sparse High-Dimensional Time Series Models. The Annals of Statistics, 43, 1535-1567.

https://doi.org/10.1214/15-AOS1315

[13] Ahelegbey, D.F., Billio, M. and Casarin, R. (2016) Sparse Graphical Vector Autoregression: A Bayesian Approach. Annals of Economics and Statistics, 123/124, 333-361. https://doi.org/10.15609/annaeconstat2009.123-124.0333

[14] Ahelegbey, D.F., Billio, M. and Casarin, R. (2016) Bayesian Graphical Models for Structural Vector Autoregressive Processes. Journal of Applied Econometrics, 31, 357-386. https://doi.org/10.1002/jae.2443

[15] Carvalho, C.M. and West, M. (2007) Dynamic Matrix-Variate Graphical Models. Bayesian Analysis, 2, 69-98. https://doi.org/10.1214/07-BA204 